\title{
Electronically Tunable Transimpedance Instrumentation Amplifier Based on OTRA
}

\author{
Rajeshwari Pandey, ${ }^{1}$ Neeta Pandey, ${ }^{1}$ and Sajal K. Paul ${ }^{2}$ \\ ${ }^{1}$ Department of Electronics and Communication Engineering, Delhi Technological University, Delhi, India \\ ${ }^{2}$ Department of Electronics Engineering, Indian School of Mines, Dhanbad, India \\ Correspondence should be addressed to Neeta Pandey; n66pandey@rediffmail.com
}

Received 31 August 2012; Revised 6 November 2012; Accepted 7 November 2012

Academic Editor: Jiun-Wei Horng

Copyright (c) 2013 Rajeshwari Pandey et al. This is an open access article distributed under the Creative Commons Attribution License, which permits unrestricted use, distribution, and reproduction in any medium, provided the original work is properly cited.

\begin{abstract}
Operational transresistance amplifier (OTRA) is the most suitable analog building block (ABB) for transimpedance type signal processing due to its very nature of current input and voltage output. In this paper, OTRA-based transimpedance instrumentation amplifier (TIA) is presented. It provides high differential gain and bandwidth, which is independent of gain. It also offers high common-mode rejection ratio (CMRR). The amplifier gain can be controlled electronically by implementing resistors using MOS transistors operating in linear region. The circuit can be made fully integrated. The proposed circuit is insensitive to parasitic input capacitances and input resistances due to the internally grounded input terminals of OTRA. Theoretical analysis is verified through PSPICE simulations and experimentation.
\end{abstract}

\section{Introduction}

An instrumentation amplifier (IA) faithfully amplifies low level differential signals in the presence of high com-monmode noise which makes it especially suitable as input stage of a signal processing system. The conventional voltage-mode instrumentation amplifiers based on operational amplifier are not capable of operating at higher frequencies because of slew rate and fixed gain-bandwidth product limitations [1]. The current mode approach has gained a lot of importance in the recent past due to its inherent wide bandwidth which is virtually independent of closed loop gain, greater linearity, and large dynamic range [1]. Thus the current-mode instrumentation amplifiers [2-4] are superior, in performance, to their voltage mode counterpart in terms of CMRR and frequency range of operation. The literature survey reveals that, currently, most of the current mode instrumentation amplifiers (CMIAs) use the second-generation current conveyor (CCII) [2-4]. These configurations are suitable for amplification of signals from voltage-source transducers. For amplification of signals from current-source transducers, TIA would be a better choice wherein the current input can be directly processed without conversion to voltage signal.
An op-amp-based TIA is presented in [5], however, it uses two current to voltage converters followed by an amplifier. OTRA being a current input voltage output $\mathrm{ABB}$ is inherently suitable for TIA implementation [6]. Therefore, in this paper an OTRA-based TIA has been proposed. It offers a high differential gain and a bandwidth which is independent of gain, unlike a traditional voltage-mode IA. It also provides high common-mode rejection ratio (CMRR).

\section{The Proposed Circuit}

OTRA is a three terminal device, shown symbolically in Figure 1, and its port relations are characterized by (1), where $R_{m}$ is the transresistance gain of OTRA. For ideal operations, $R_{m}$ approaches infinity and forces the input currents to be equal. Thus, OTRA must be used in a negative feedback configuration $[7,8]$. It is also free from parasitic input capacitances, and

$$
\left[\begin{array}{c}
V_{p} \\
V_{n} \\
V_{O}
\end{array}\right]=\left[\begin{array}{rrr}
0 & 0 & 0 \\
0 & 0 & 0 \\
R_{m} & -R_{m} & 0
\end{array}\right]\left[\begin{array}{c}
I_{p} \\
I_{n} \\
I_{O}
\end{array}\right]
$$




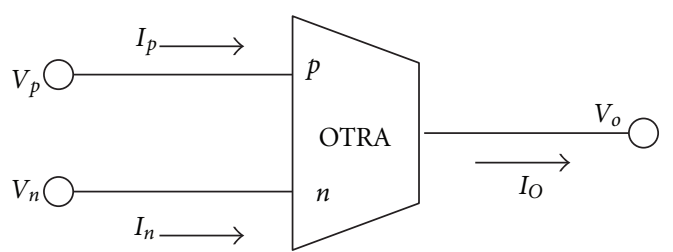

Figure 1: OTRA Circuit symbol.

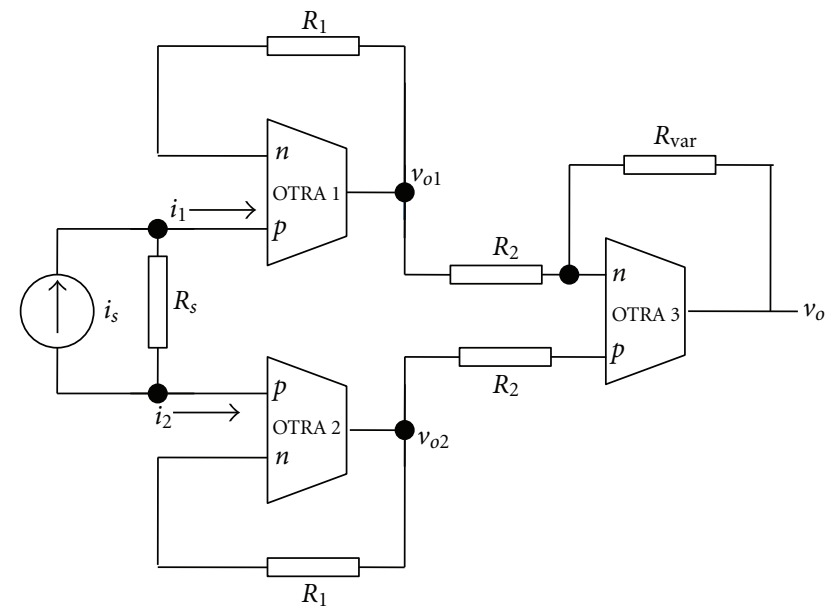

Figure 2: The proposed TIA.

resistances as its input terminals are virtually grounded, and, hence, nonideality problem is less in circuits implemented with OTRA. Figure 2 shows the proposed circuit. It consists of three OTRAs and five resistors. The differential transimpedance gain $\left(A_{d}\right)$ for the amplifier can be computed as follows:

$$
\begin{gathered}
v_{o 1}=i_{1} R_{1}, \\
v_{o 2}=i_{2} R_{1}, \\
A_{d}=\frac{v_{0}}{i_{2}-i_{1}}=\frac{R_{\mathrm{var}} R_{1}}{R_{2}} .
\end{gathered}
$$

It can be seen from (4) that $A_{d}$ can be varied by varying the resistance $R_{\mathrm{var}}$.

Ideally the transresistance gain $R_{m}$ is assumed to approach infinity. However, practically $R_{m}$ is a frequency dependent finite value [8]. Considering a single pole model for the transresistance gain, $R_{m}$ can be expressed as

$$
R_{m}(s)=\left(\frac{R_{0}}{1+s / \omega_{0}}\right)
$$

where $R_{0}$ is low frequency transresistance gain, and $\omega_{0}$ is the pole angular frequency of the OTRA.

For high frequency applications, the transresistance gain $R_{m}(s)$ reduces to

$$
R_{m}(s) \approx\left(\frac{1}{s C_{p}}\right)
$$

Taking this effect into account, output voltages at different nodes are given as

$$
\begin{aligned}
& v_{o 1}=\frac{i_{1} R_{1}}{1+s C_{P 1} R_{1}}, \\
& v_{o 2}=\frac{i_{2} R_{1}}{1+s C_{P 2} R_{1}},
\end{aligned}
$$

$$
v_{0}=\frac{R_{1} R_{\mathrm{var}}}{R_{2}\left(1+s C_{P 3} R_{\mathrm{var}}\right)}\left(\frac{i_{2}}{\left(1+s C_{P 1} R_{1}\right)}-\frac{i_{1}}{\left(1+s C_{P 2} R_{1}\right)}\right) \text {, }
$$

where $C_{p 1}, C_{p 2}$, and $C_{p 3}$ are the parasitic capacitances of OTRA1, OTRA2, and OTRA3, respectively.

Considering $C_{p 1}=C_{p 2}$ in (9), the $A_{d}$ of the circuit given by (4) modifies to

$$
A_{d}=\frac{v_{0}}{i_{2}-i_{1}}=\frac{R_{\mathrm{var}} R_{1}}{R_{2}} \varepsilon_{\mathrm{uc}}(s),
$$

where

$$
\varepsilon_{\mathrm{uc}}(s)=\frac{1}{\left(1+s C_{P 1} R_{1}\right)\left(1+s C_{P 3} R_{\mathrm{var}}\right)}
$$

is the uncompensated error function.

From (9), it is to be noticed that the gain $A_{d}$ can be adjusted by $R_{\mathrm{var}} R_{1} / R_{2}$, and the bandwidth is controlled by $\varepsilon_{\mathrm{uc}}(s)$. Thus, the gain of the amplifier can be adjusted by varying $R_{\mathrm{var}}$ without affecting the bandwidth (BW).

From (9), by using $i_{1}=i_{2}=i_{c}$, the transimpedance common-mode gain $\left(A_{c}\right)$ can be computed as

$$
\begin{aligned}
A_{c}=\frac{v_{0}}{i_{c}}= & \frac{R_{1} R_{\mathrm{var}}}{R_{2}\left(1+s C_{P 3} R_{\mathrm{var}}\right)} \\
& \times\left(\frac{1}{\left(1+s C_{P 2} R_{1}\right)}-\frac{1}{\left(1+s C_{P 1} R_{1}\right)}\right) .
\end{aligned}
$$

The performance parameter CMRR which defined as the ratio of differential gain to common-mode gain can be computed as

$$
\mathrm{CMRR}=\frac{A_{d}}{A_{c}}=\frac{2+s\left(C_{p 1}+C_{p 2}\right) R_{1}}{s\left(C_{P 1}-C_{p 2}\right) R_{1}} .
$$

For high frequency applications, compensation methods must be employed to account for the error $\varepsilon_{\mathrm{uc}}(s)$ introduced in (4) and given by (11). High frequency passive compensated topology of the TIA is shown in Figure 3. Routine analysis of Figure 3 results in

$$
A_{d}=\frac{v_{0}}{i_{2}-i_{1}}=\frac{R_{\mathrm{var}} R_{1}}{R_{2}} \varepsilon_{c}(s)
$$

where

$$
\varepsilon_{c}(s)=\frac{1}{\left(1+R_{1} s\left(C_{P 1}-C_{1}\right)\right)\left(1+R_{\mathrm{var}} s\left(C_{P 3}-C_{3}\right)\right)}
$$

is the compensated error function. 


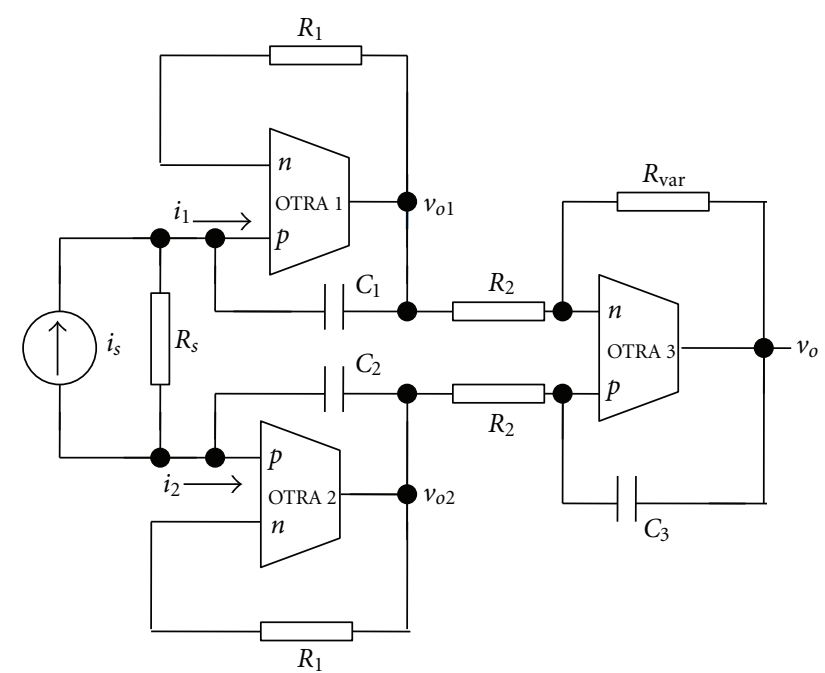

FIGURE 3: TIA with high frequency compensation.

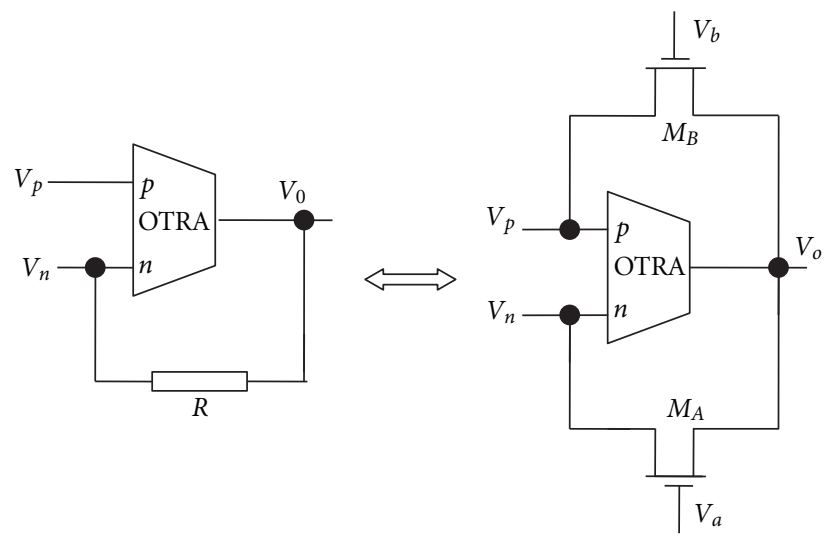

FIGURE 4: MOS implementation of a linear resistance connected between negative input and output terminals of OTRA.

By taking $C_{1}=C_{2}=C_{p 1}$ and $C_{3}=C_{p 3}, \varepsilon_{c}(s)$ reduces to 1 , which makes (14) the same as (4). The effect of single pole model of $R_{m}$ can thus be eliminated.

It is well know that the linear passive resistor consumes a large chip area as compared to the linear resistor implementation using transistors operating in nonsaturation region. The differential input of OTRA allows the resistors connected to the input terminals of OTRA to be implemented using MOS transistors with complete nonlinearity cancellation [8]. Figure 4 shows a typical MOS implementation of resistance connected between negative input and output terminals of OTRA. The resistance value may be adjusted by appropriate choice of gate voltages of these transistors thereby making gain of TIA electronically tunable. The value of resistance so obtained is expressed as

$$
R=\frac{1}{\mu_{n} C_{\text {ox }}(W / L)\left(V_{a}-V_{b}\right)},
$$

where $\mu_{n}, C_{o x}, W$, and $L$ are electron mobility, oxide capacitance per unit gate area, effective channel width, and effective

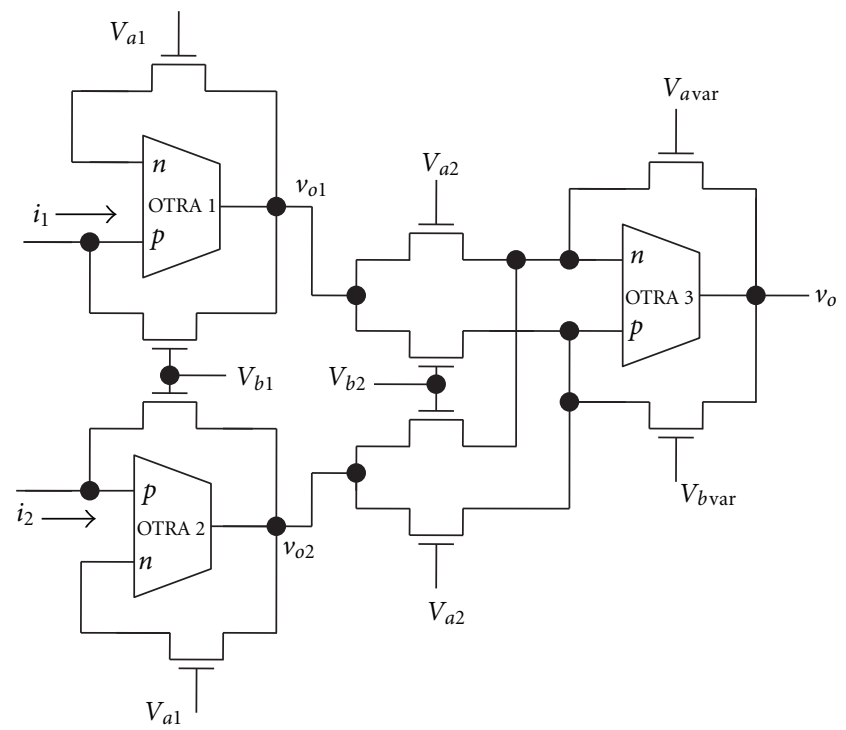

FIGURE 5: MOS-based implementation of the proposed TIA.

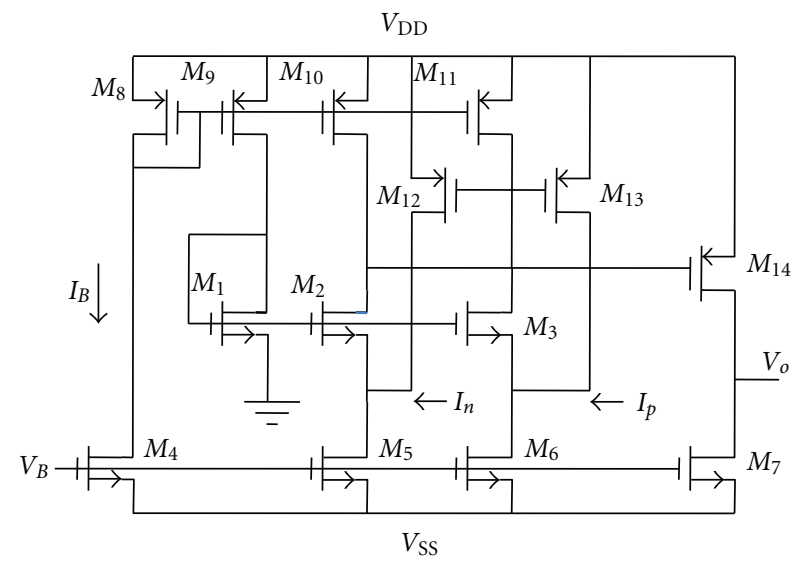

FIGURE 6: CMOS implementation of OTRA [9].

channel length of MOS, respectively. $V_{a}$ and $V_{b}$ are the gate voltages. Thus, MOS transistor implementation of $R_{1}, R_{2}$, and $R_{\mathrm{var}}$ not only makes the TIA gain electronically tunable but also makes the circuit suitable for integration. Figure 5 shows the MOS-based implementation of the proposed TIA.

\section{Simulation and Experimental Results}

The performance of the proposed TIA is verified through SPICE simulation using $0.5 \mu \mathrm{m}$ CMOS process parameters provided by MOSIS (AGILENT). CMOS implementation of the OTRA [9] shown in Figure 6 is used for simulation. Aspect ratios used for different transistors are the same as in [9] and are given in Table 1. The taken supply voltages are $\pm 1.5 \mathrm{~V}$.

Figure 7(a) shows the frequency response of the proposed amplifier. The input differential current was chosen as $5 \mathrm{~mA}$. The component values are taken as $R_{1}=5 \mathrm{~K} \Omega$ and $R_{2}=$ $1 \mathrm{~K} \Omega . R_{\mathrm{VAR}}$ is assigned values as $10 \mathrm{~K} \Omega, 5 \mathrm{~K} \Omega$, and $0.8 \mathrm{~K} \Omega$, 


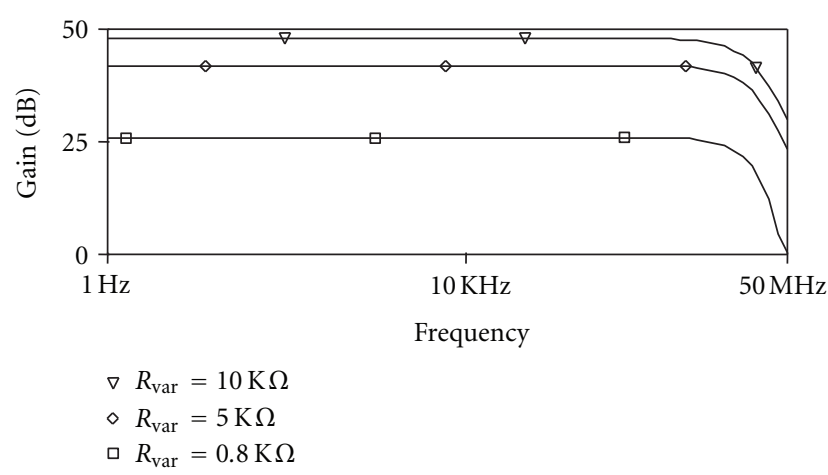

(a)

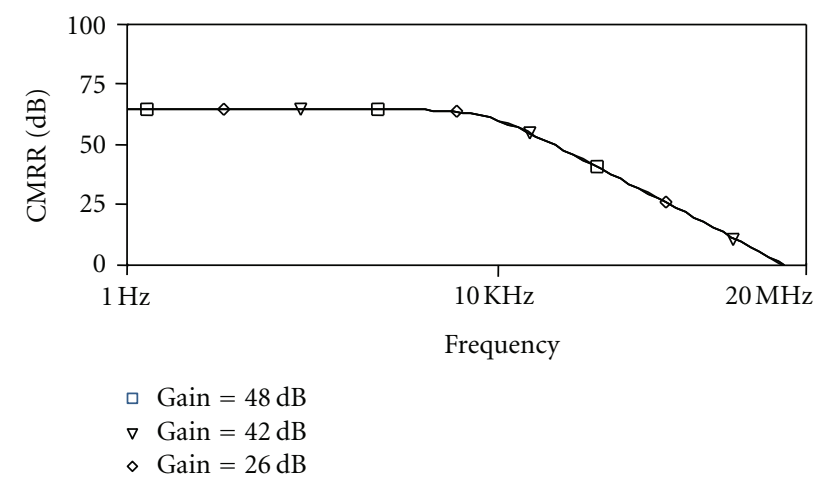

(b)

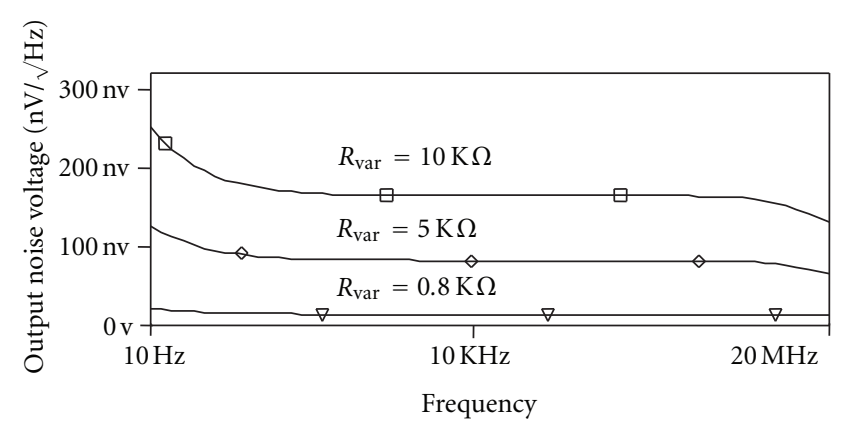

(c)

FIGURE 7: (a) Frequency response of the proposed TIA, (b) CMRR response of the proposed TIA, and (c) output noise spectral density of the proposed TIA.

TABLE 1: Aspect ratio of the transistors in OTRA circuit.

\begin{tabular}{lc}
\hline Transistor & $W(\mu \mathrm{m}) / L(\mu \mathrm{m})$ \\
\hline M1-M3 & $100 / 2.5$ \\
M4 & $10 / 2.5$ \\
M5, M6 & $30 / 2.5$ \\
M7 & $10 / 2.5$ \\
M8-M11 & $50 / 2.5$ \\
M12, M13 & $100 / 2.5$ \\
M14 & $50 / 0.5$ \\
\hline
\end{tabular}

the differential gains obtained were $48 \mathrm{~dB}, 42 \mathrm{~dB}$, and $26 \mathrm{~dB}$, respectively. The $3 \mathrm{~dB}$ frequency of the amplifier is $10 \mathrm{MHz}$

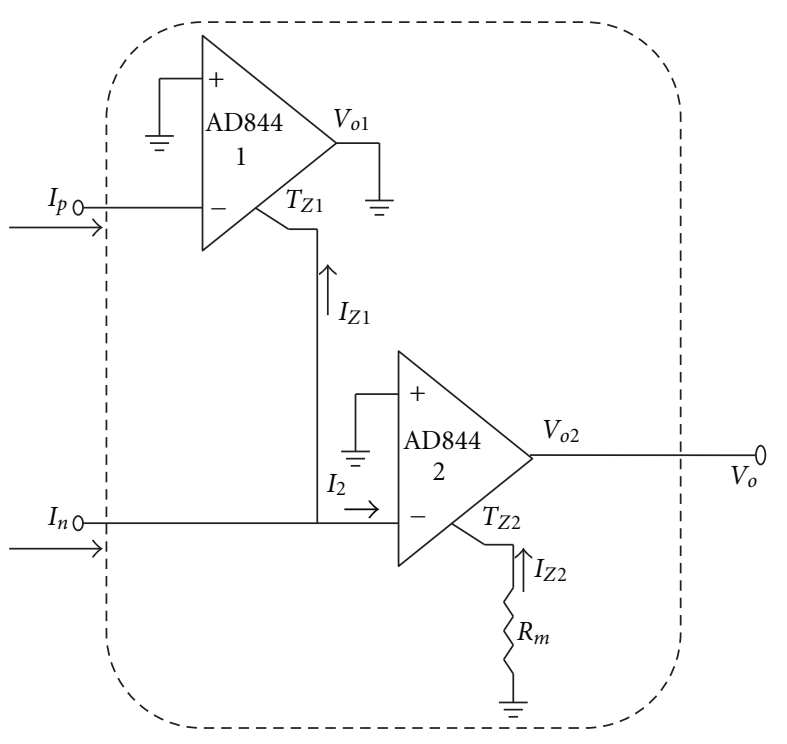

FIgURE 8: OTRA realization using commercial IC AD 844AN [10].

for all the three cases confirming that the bandwidth of the amplifier is independent of gain. Total power consumption of the proposed TIA is simulated to be $4.93 \mathrm{~mW}$.

Figure 7(b) shows the CMRR response of the circuit for differential gains of 26,42 , and $48 \mathrm{~dB}$. It is observed that the proposed TIA exhibits a CMRR magnitude of $64.5 \mathrm{~dB}$ and bandwidth of $10 \mathrm{KHz}$, which is independent of gain.

The simulation results of the noise performance analysis of the proposed TIA are depicted in Figure 7(c). The component values are chosen to be the same as for differential gain. It may be noticed from the Figure $7(\mathrm{c})$ that the noise level being low would result in high signal-to-noise ratio of the TIA.

A hardware prototype of the proposed TIA is also designed to test its functionality experimentally. The OTRA is realized using commercial IC AD 844AN [10] as shown in Figure 8. Supply voltages used are $\pm 5 \mathrm{~V}$. The experimental and simulated frequency response for the TIA, for $R_{1}=5 \mathrm{~K} \Omega$, $R_{2}=1 \mathrm{~K} \Omega$, and $R_{\mathrm{VAR}}=10 \mathrm{~K} \Omega$, are shown in Figure 9 (a), and CMRR response is shown in Figure 9(b). The slight variations in experimental and simulated results may be due to tolerance of the component values. Observed outputs showing the performance of the proposed TIA at $70 \mathrm{KHz}$ and $2 \mathrm{MHz}$ for $R_{1}=5 \mathrm{~K} \Omega, R_{2}=1 \mathrm{~K} \Omega$, and $R_{\mathrm{VAR}}=10 \mathrm{~K} \Omega$ are given in Figure 9(c).

\section{Conclusion}

An OTRA-based TIA is presented which is suitable for amplification of signals from current-source transducers. The circuit provides high gain for a wide range of frequencies, and the bandwidth of the amplifier is independent of its gain. The proposed circuit, to the best knowledge of authors, is the only current mode TIA. A voltage mode op-amp-based transresistance instrumentation amplifier is proposed in [5] which uses three op-amps and nine resistors, a quite large number as compared to the proposed TIA. Also, the amplifier of [5] 


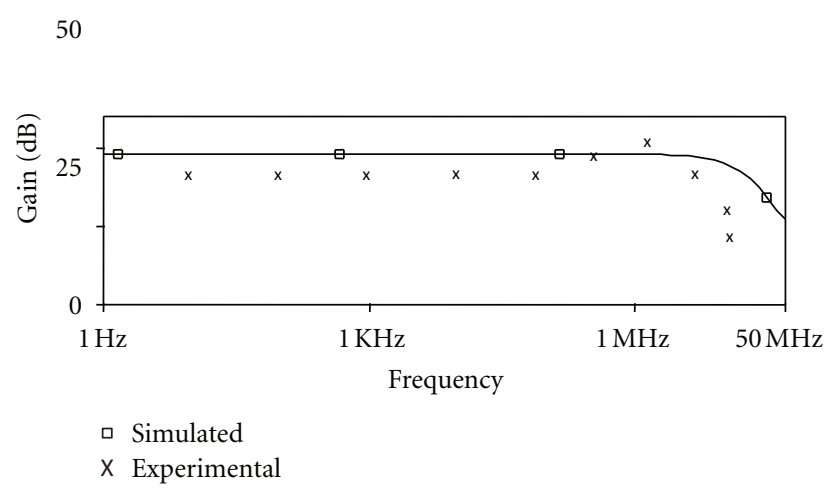

(a) Frequency response for $R_{\mathrm{VAR}}=10 \mathrm{~K} \Omega$

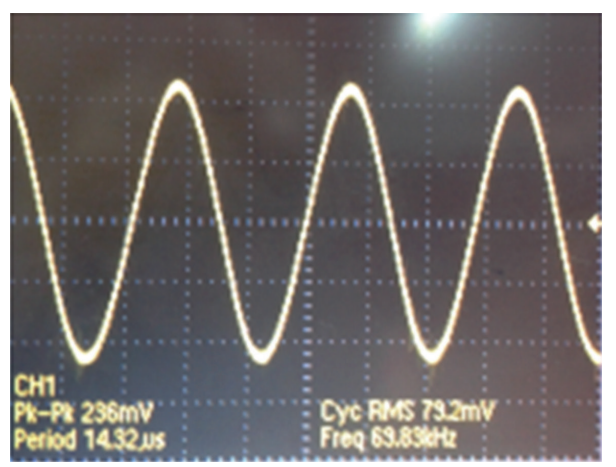

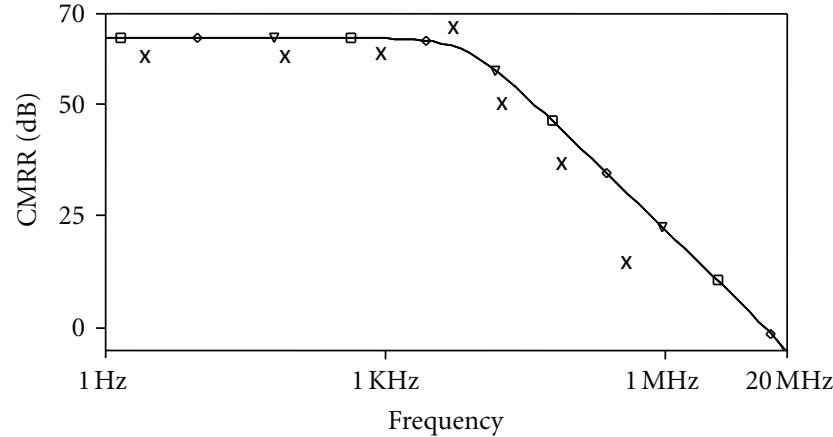

$\because$ Simulated

$x \times x$ Experimental

(b) CMRR response

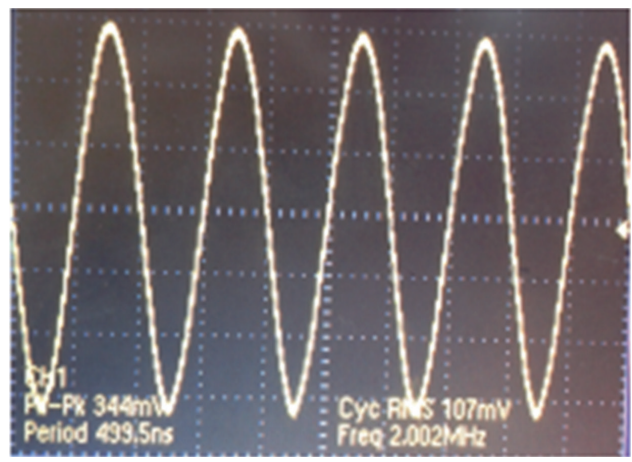

(c) Observed output for $70 \mathrm{KHz}$ input and observed output for $2 \mathrm{MHz}$ input

FIGURE 9: Experimental results of the proposed TIA.

was designed, for a specific low frequency application in a gamma-ray dosimeter, and its other performance parameters are not available for comparison.

The gain of the proposed amplifier can be electronically tuned by implementing the passive resistor using MOS transistor. This also makes the circuit fully integrated. The proposed circuit can easily be compensated for parasitics. It provides advantages of current mode design techniques and at the same time provides voltage output suitable for driving existing voltage mode circuits. Theoretical propositions are verified through PSPICE simulations and experimental results.

\section{References}

[1] C. Toumazou, F. J. Lidgey, and D. G. Haigh, Analogue IC Design: The Current Mode Approach, chapter 1, Peregrinus, Stevenage, UK, 1990.

[2] Q. S. Zhu, F. J. Lidgey, and W. J. Su, "High CMRR, second generation current-mode instrumentation amplifiers," in Proceedings of the IEEE International Symposium on Circuits and Systems, vol. 2, pp. 1326-1328, May 1993.

[3] K. Koli and K. A. I. Halonen, "CMRR enhancement techniques for current-mode instrumentation amplifiers," IEEE Transactions on Circuits and Systems I, vol. 47, no. 5, pp. 622-632, 2000.
[4] Y. H. Ghallab, W. Badawy, K. V. I. S. Kaler, and B. J. Maundy, "A novel current-mode instrumentation amplifier based on operational floating current conveyor," IEEE Transactions on Instrumentation and Measurement, vol. 54, no. 5, pp. 1941-1949, 2005.

[5] D. C. Agouridis and R. J. Fox, "Transresistance instrumentation amplifier," Proceedings of the IEEE, vol. 66, no. 10, pp. 1286-1287, 1978.

[6] S. Kilinç and U. Çam, "Transimpedance type fully integrated biquadratic filters using operational transresistance amplifiers," Analog Integrated Circuits and Signal Processing, vol. 47, no. 2, pp. 193-198, 2006.

[7] J. J. Chen, H. W. Tsao, and C. C. Chen, "Operational transresistance amplifier using CMOS technology," Electronics Letters, vol. 28, no. 22, pp. 2087-2088, 1992.

[8] K. N. Salama and A. M. Soliman, "CMOS operational transresistance amplifier for analog signal processing," Microelectronics Journal, vol. 30, no. 3, pp. 235-245, 1999.

[9] H. Mostafa and A. M. Soliman, "A modified CMOS realization of the operational transresistance amplifier (OTRA)," Frequenz, vol. 60, no. 3-4, pp. 70-76, 2006.

[10] Y. K. Lo and H. C. Chien, "Current-mode monostable multivibrators using OTRAs," IEEE Transactions on Circuits and Systems II, vol. 53, no. 11, pp. 1274-1278, 2006. 

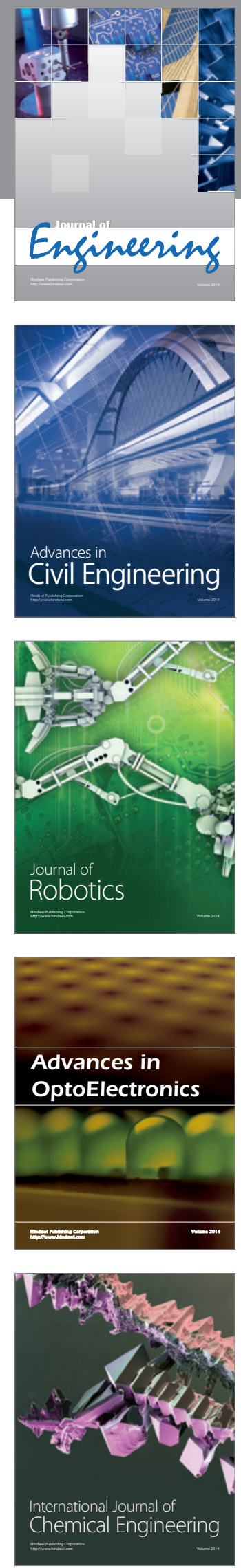

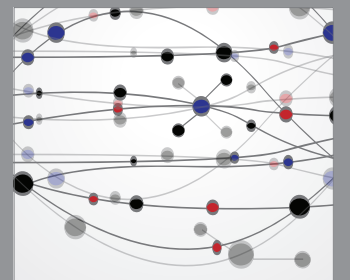

The Scientific World Journal
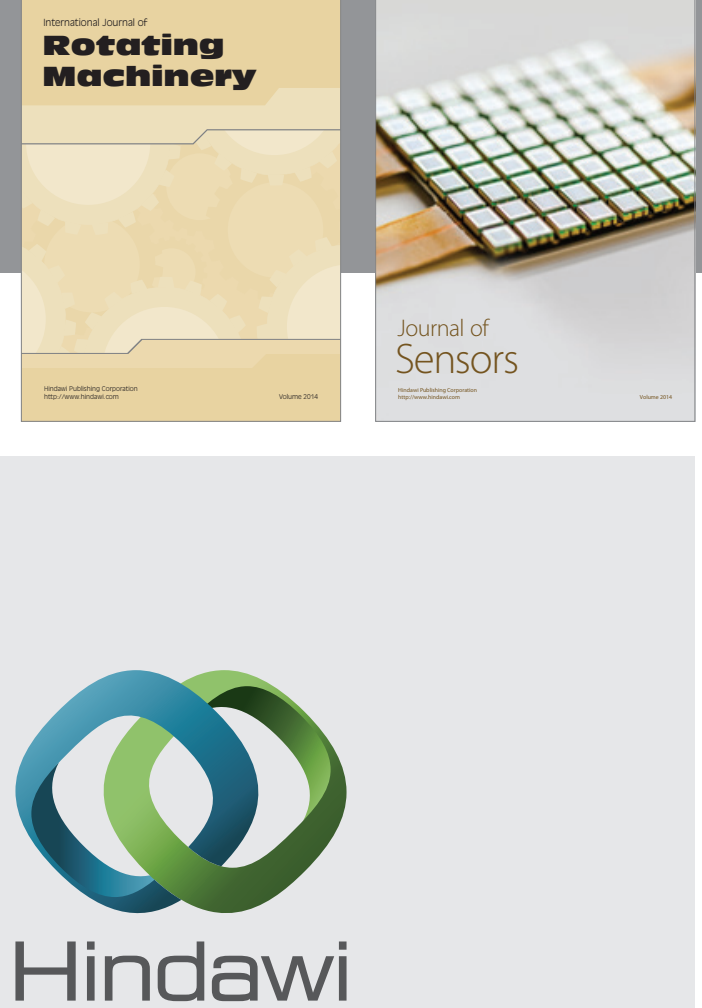

Submit your manuscripts at http://www.hindawi.com
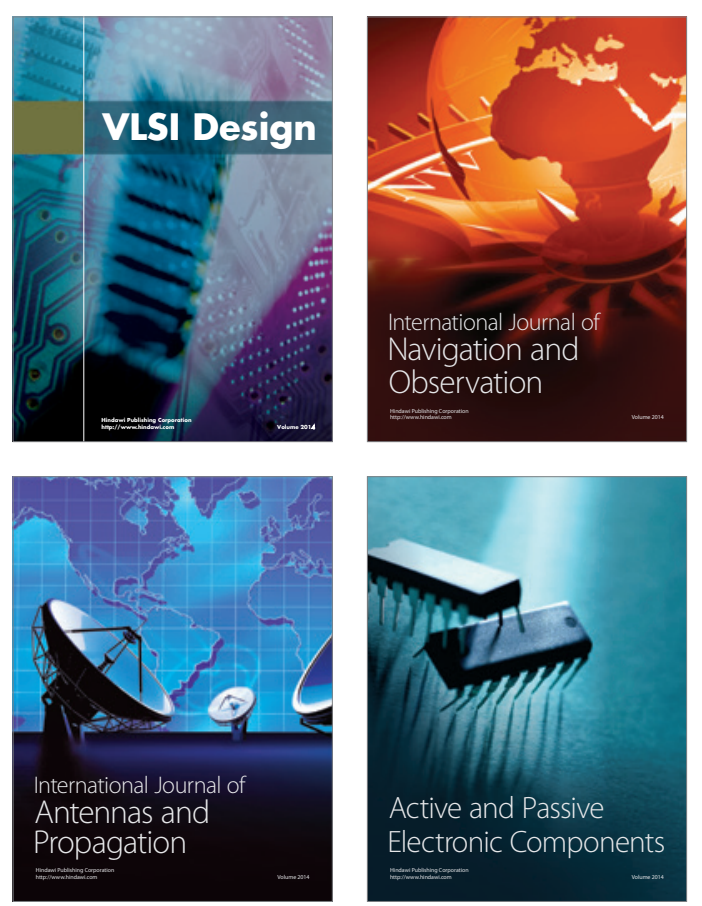
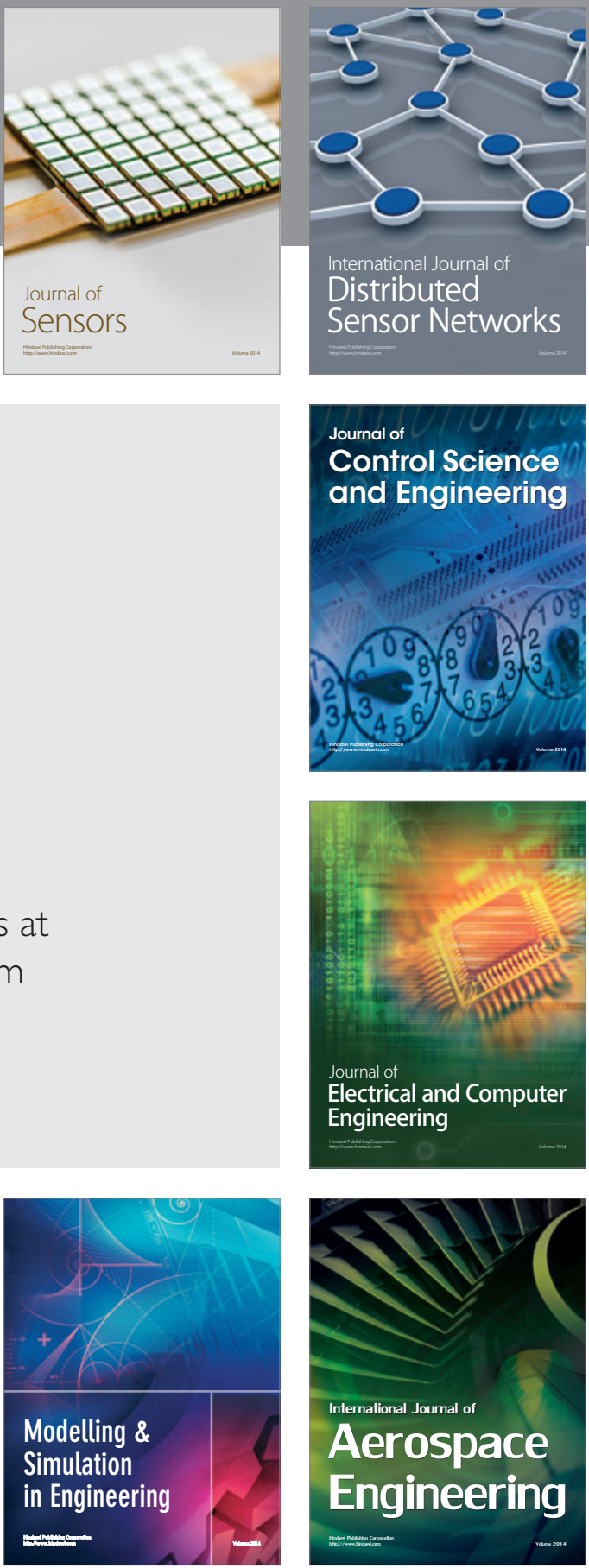

Journal of

Control Science

and Engineering
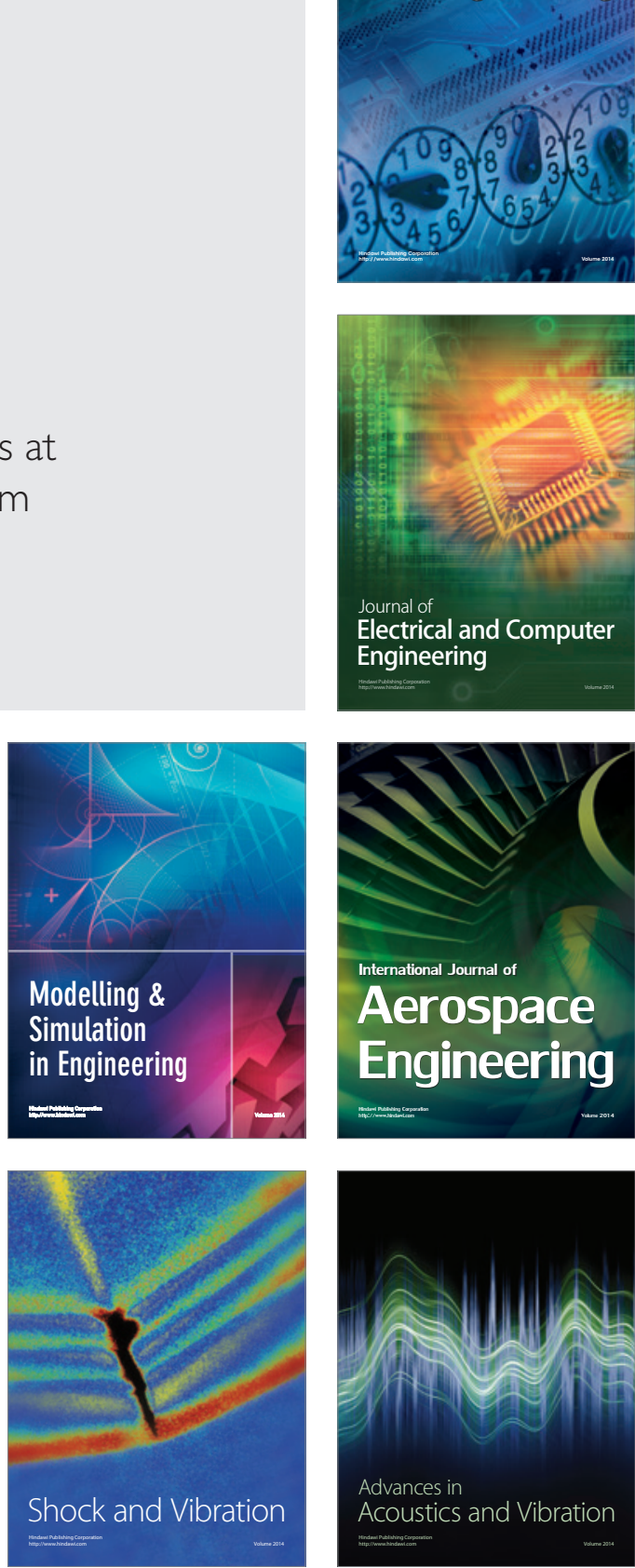